\title{
Grain-Subgrain Structure and Vacancy-Type Defects in Submicrocrystalline Nickel at Low Temperature Annealing
}

\author{
P.V. KuZnetsov ${ }^{a, b, *}$, A.M. LideR ${ }^{a}$, Yu.S. BORduleV ${ }^{a}$, R.S. LAPteV ${ }^{a}$, T.V. \\ RAKHMATULINA $^{b}$ AND A.V. KORZNIKOV ${ }^{c}$ \\ ${ }^{a}$ National Research Tomsk Polytechnic University, Tomsk, Russia \\ ${ }^{b}$ Institute of Strength Physics and Materials Science, Siberian Branch of RAS, Tomsk, Russia \\ ${ }^{c}$ Institute for Metals Superplasticity Problems of Russian Academy of Sciences, Ufa, Russia
}

\begin{abstract}
Scanning tunneling microscopy, positron annihilation and X-ray diffraction were applied for the study of annealing of submicrocrystalline nickel prepared by equal channel angular pressing. Several processes were revealed in the structure of submicrocrystalline nickel on different scale levels during annealing in the range $\Delta T=(20 \div$ $360)^{\circ} \mathrm{C}$. A decrease of grain nonequiaxiality and further structure refinement were observed with a temperature increase in the range $\Delta T=(20 \div 180)^{\circ} \mathrm{C}$. Subgrain growth with maximum $=60 \mathrm{~nm}$ at $120^{\circ} \mathrm{C}$ occurred on the lower scale level within the same temperature range. Grain growth and microstress decrease in submicrocrystalline nickel observed at $T>180^{\circ} \mathrm{C}$ indicate the beginning of recrystallization. The main positron trap centers were identified in submicrocrystalline nickel within different temperature ranges. In as-prepared samples positrons are trapped at dislocation-type defects and vacancy clusters that can include up to 5 vacancies. At the annealing temperature $\Delta T=(20 \div 180)^{\circ} \mathrm{C}$ positrons are trapped at low-angle boundaries enriched by impurities. Within the range $\Delta T=(180 \div 360)^{\circ} \mathrm{C}$ the dominant trap is dislocations.
\end{abstract}

DOI: $10.12693 /$ APhysPolA.128.714

PACS: 68.37.Ef, 61.72.J-, 61.72.Lk, 61.72.-y, 81.40.Ef, 78.70.Bj

\section{Introduction}

Grain refinement of material structure down to $\mu \mathrm{m}$ size by means of severe plastic deformation (SPD) can lead to new unique physical and mechanical properties of material in comparison with their coarse-grain analogies [1, 2]. Submicrocrystalline (SMC) materials, in particular, have high yield strength and microhardness which are often achieved without essential loss of plasticity and therefore they are of direct practical interest $[1,2]$.

During SPD part of energy is accumulated as hierarchy of defects of different dimensions: boundaries of various types, dislocations, vacancies $[1,2]$. To decrease the degree of non-equilibrium and to stabilize structure SMC materials are usually subjected to low-temperature annealing in order to prevent significant grain growth and deterioration of mechanical properties [1]. Release of excess energy occurs during SMC materials heating via boundaries transformation, dislocations rearrangement and annihilation, and annealing of defects in bulk crystallites. Study of the processes is of great interest for understanding the role of various defects in the SMC materials properties formation during annealing. It requires several methods of grain-subgrain structure (GSS) and point defects study in SMC materials. This work aims at studying the GSS and vacancy-type defects evolution in SMC low-temperature annealed nickel using scanning

*corresponding author; e-mail: kpv@ispms.tsc.ru tunneling microscopy (STM), positron annihilation (PA) and X-ray diffraction (XRD).

\section{Materials and methods}

A nickel ingot of $99.998 \%$ purity was annealed at $500{ }^{\circ} \mathrm{C}$ during $1 \mathrm{~h}$. The average grain size was about $45 \mu \mathrm{m}$. SMC structure was produced by equal-channel angular pressing (ECAP) implementing route $\mathrm{Bc}(N=4$ passes) at room temperature, followed by rolling up to $400 \%$ reduction. The samples were cut out by electrospark method across the rolling direction and then mechanically polished.

An isochronal annealing was held in argon ambience for $15 \mathrm{~min}$ at temperature range $\Delta T=(20 \div 360)^{\circ} \mathrm{C}$. Several samples were recrystallized at $800^{\circ} \mathrm{C}$ for $1 \mathrm{~h}$. GSS was revealed by means of electrochemical polishing and subsequent etching.

Samples GSS was studied using scanning tunnel microscope operating in constant current mode. STM data format was $300 \times 300$ pixels with 256 gradations in height. The technique of GSS element sizes and grain boundaries energy estimation was described in detail in [3].

XRD analysis of samples was performed with X-ray diffractometer DRON-7 ("NANOTECH" of ISPMS SB RAS) with a Co $K_{\alpha}$ source, using symmetric reflection scheme without a monochromator. The coherentscattering region (CSR) size and microstrain values were derived from integral diffraction peaks widths by using the Williamson-Hall method.

PA was studied by measuring positron lifetime spectra (PLS) and the Doppler broadening (DB) of annihilation 
line. PA spectrometers implemented in this work are described in detail in [4].

\section{Results and discussions}

STM images $1800 \times 1800 \mathrm{~nm}^{2}, 880 \times 880 \mathrm{~nm}^{2}$, and $440 \times 440 \mathrm{~nm}^{2}$ from surface samples were obtained to study GSS of SMC nickel. The relative number of grains in smaller STM images decreases and the relative proportion of subgrain structure resolved by STM increases. Therefore, analysis of the GSS element sizes at different scale levels allows one to reveal processes occurring in grain and subgrain structures with increasing temperature.

Typical patterns of the GSS at different scales in asprepared and annealed at $=240{ }^{\circ} \mathrm{C}$ samples are shown at Fig. 1a,b, respectively. One can see (Fig. 1b) that annealing at $=240{ }^{\circ} \mathrm{C}$ leads to grain growth of SMC nickel.

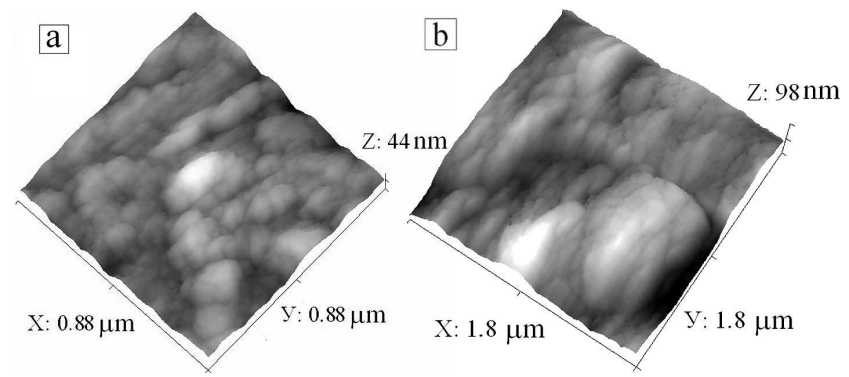

Fig. 1. STM images of the SMC nickel grain structure in as-prepared state a) and annealed at $240^{\circ} \mathrm{C}$ b).

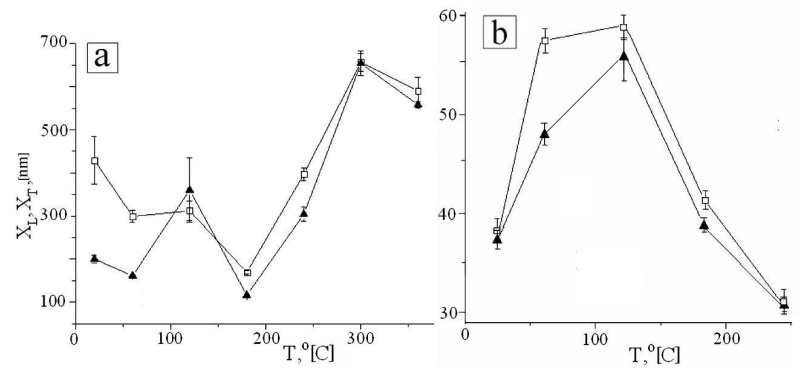

Fig. 2. The dependencies of average GSS sizes along $\left\langle X_{L}\right\rangle$ (ם) and across $\left\langle X_{T}\right\rangle(\boldsymbol{\Delta})$ rolling on annealing temperature at different scales: a) $1800 \times 1800 \mathrm{~nm}$; b) $440 \times 440 \mathrm{~nm}$.

Histograms of GSS sizes distribution along and across rolling direction were obtained using STM data and described by lognormal function.

Corresponding dependence of average GSS sizes on temperature are shown in Fig. 2. Several processes were identified in SMC nickel structure at different scale levels at the temperature ranges $\Delta T=(20 \div 360)^{\circ} \mathrm{C}$ (Fig. 2a,b).

In as-prepared samples average GSS sizes along rolling $\left(\left\langle X_{L}\right\rangle\right)$ are greater than sizes across rolling $\left(\left\langle X_{T}\right\rangle\right)$ (Fig. 2a) that indicates structure non-equiaxiality.
An increase in the annealing temperature up to $120^{\circ} \mathrm{C}$ leads to lower non-equiaxiality of grains. Another process in the SMC nickel at the temperature range $\Delta T=$ $(20 \div 180)^{\circ} \mathrm{C}$ is grain refinement up to average grain size $D=(130-170) \mathrm{nm}$ (Fig. 2a). We suppose that nonequiaxial grains, formed after ECAP and followed rolling (Fig. 1a), have substantial fraction of low-angle boundaries (LAB) across the long axis (LA). During heating the mechanical equilibrium of dislocation structure is upset and leads to dislocation rearrangement, an increase of the LAB misorientation across the LA grain and new high-angle boundaries (HAB) formation. This reduces grains non-equiaxiality and leads to further grain refinement. The effect of grain refinement during the dynamical heating of SMC nickel produced by 4 passes of high pressure torsion (HPT) was also observed in [5] where possible explanation was suggested.

On the lower scale level subgrain growth took place with a maximum at $120^{\circ} \mathrm{C}$ (Fig. $2 \mathrm{~b}$ ) that reduced the excess of crystals energy and correlated with the change of CSR sizes. The subgrain size decrease at $T \geq 180^{\circ}$ (Fig. 2b) is probably an apparent effect associated with SMC structure heterogeneity. The proximity of sizes $\left\langle X_{L}\right\rangle,\left\langle X_{T}\right\rangle$ at $T \geq 180^{\circ}$ to the appropriate ones for as-prepared samples (Fig. 2b) might indicate that the subgrains were not transformed at annealing in certain sites of SMC structure due to the low value of the driving force associated with the dislocations distribution.

Annealing at $T>180^{\circ} \mathrm{C}$ leads to grain growth (Fig. 2a), which is directly observed by STM (Fig. 1b). In addition, at $T>240^{\circ} \mathrm{C}$ samples microhardness and crystal structure microstresses decreased. The data clearly indicate the beginning of recrystallization in SMC nickel.

XRD analysis reveals pronounced samples deformation texture that does not change significantly during annealing within the temperature range $\Delta T=(20 \div 360)^{\circ} \mathrm{C}$. Therefore the SMC nickel recrystallization at $T \geq 240^{\circ} \mathrm{C}$ is not associated with appearance of grains with new orientation. We assume the new microstructure to be formed without migrating $\mathrm{HAB}$, i.e. so-called recrystallization in situ takes place. It was recently detected by authors of [6] for SMC nickel samples prepared by ECAP.

Analysis of PLS of as-prepared and annealed at $\Delta T=$ $(60 \div 360)^{\circ} \mathrm{C}$ samples enabled detection of components with specific lifetimes $\tau_{2}=(150 \div 165)$ ps and $\tau_{3}=$ $(230 \div 290)$ ps. Dependence of $\tau_{2}(\circ)$ and $\tau_{3}(\square)$ and corresponding intensities $I_{2}(\circ)$ and $I_{3}(\square)$ on the annealing temperature are depicted in Fig. 3a and b. Within the temperature range $\Delta T=(20 \div 240)^{\circ} \mathrm{C}$ the component $\tau_{2}$ decreases from 165 to $150 \mathrm{ps}$ (Fig. 3a), that corresponds to the lifetime of positrons trapped at dislocations in nickel [7]. The intensity $I_{2}$ of this component varies from 87 to $95 \%$ at $\Delta T=(60 \div 360)^{\circ} \mathrm{C}$ (Fig. 3b).

The component $\tau_{3}=(230 \div 290)$ ps indicate positrons trap at defects with larger free volume in comparison to single vacancies and dislocations [7-9]. According to Refs. [7-9] the values $\tau_{3}=(230 \div 290)$ ps are considered to be typical for vacancy clusters. Using the positron 

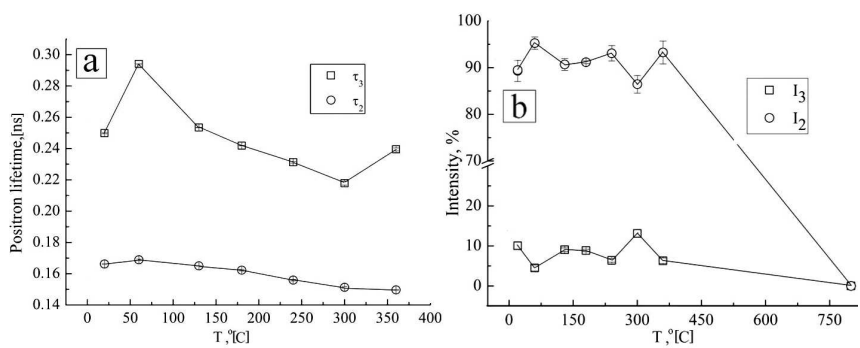

Fig. 3. Lifetime of the components resolved in PLS $\tau_{2}$, $\tau_{3}$ (a) and corresponding intensities $\mathrm{I}_{2}$ and $\mathrm{I}_{3}$ (b) as a function of annealing temperature.

lifetime theoretical dependence on the vacancy cluster size [10] we estimate their sizes in SMK nickel samples to vary from 4 to 9 vacancies at $\Delta T=(20 \div 360)^{\circ} \mathrm{C}$. The component $I_{3}$ intensity varies from 5 to $14 \%$. The similar effect of vacancy clusters size alteration during annealing of SMC nickel produced by HPT was observed in [7].

DB spectra of annihilation line allow studying electron momentum distribution [11]. Analysis of DB spectra of annihilation line involves $S$ and $W$ parameters corresponding to annihilation of positrons with valence and core electrons, respectively. Parameters $S$ and $W$ are defined as the ratio of annihilation events number in center/wings of distribution plot to the area of the annihilation peak $511 \mathrm{keV}$, respectively [11]. When positrons are trapped at vacancy defects the probability of their annihilation with valence electrons possessing low values of momentum increases, while the probability of annihilation with high momentum core electrons decreases. This leads to growing $S$ and decreasing $W$, which is also affected by chemical environment at the site of PA.

The parameters $S$ and $W$ depend on defect concentration as well as type [11]. Authors of Ref. [12] have suggested graphic method for determination of the defect parameter $R$, which does not depend on defect concentration, but is determined only by defect type. According to [12], the incline of a straight line of the function $S=f(W)$ plotted using experimentally measured values of $S_{d}, W_{d}$ for a series of samples yields the parameter value of $R$ for the defect which is the dominant positron trap.

Thus, the change of an inclination of straight line $S=f(W)$ dependence means the change of the parameter $R$ and a dominant positron trapping defect.

Figure 4 shows the normalized dependences $S / S_{b}$ and $W / W_{b}$ on annealing temperature, where $S_{b}, W_{b}$ are the values of parameters for recrystallized defect-free samples. High values of $S$-parameter and low values of $W$ parameter in as-prepared SMC nickel samples (Fig. 4) indicate the positron trapping at vacancy defects. Decrease of $S$-parameter at $\Delta T=(120 \div 240)^{\circ} \mathrm{C}$ indicates the annealing of vacancy defects. Compared to $S$-parameter, $W$-parameter starts changing at lower annealing temperatures $\Delta T=(20 \div 180)^{\circ} \mathrm{C}$ (Fig. 4).

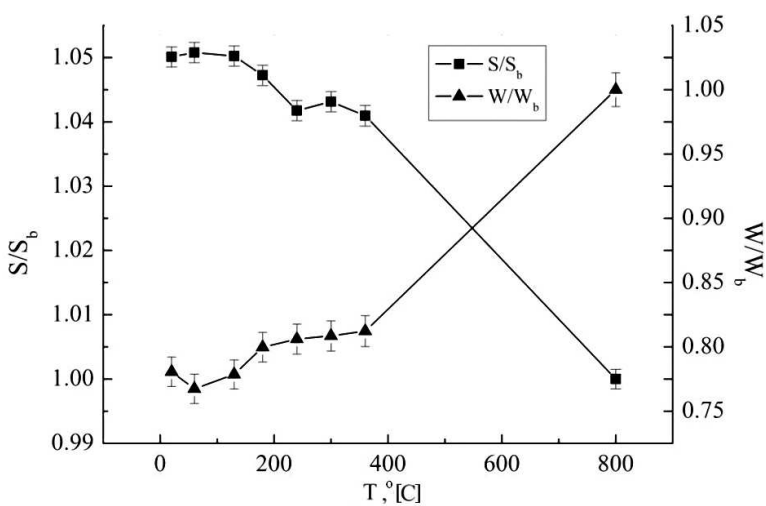

Fig. 4. Dependence of $S$ and $W$ parameters on SMC nickel annealing temperature. Parameters are respectively normalized to $\mathrm{S}_{b}, \mathrm{~W}_{b}$ for recrystallized samples.

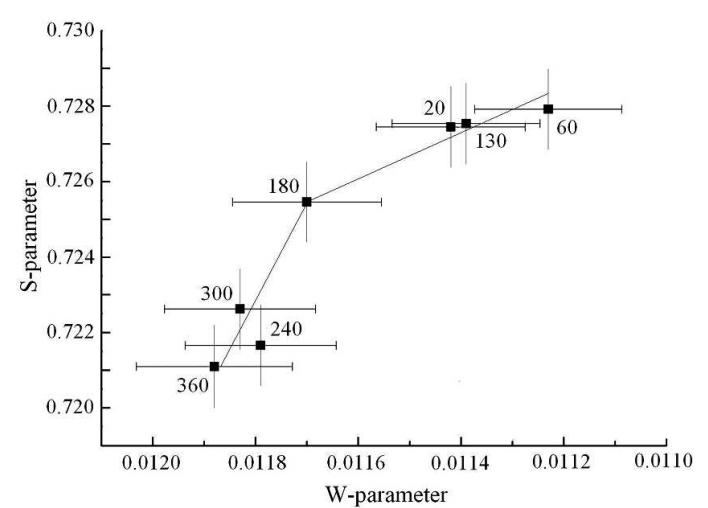

Fig. 5. Dependence of S-parameter on W-parameter for SMC nickel samples annealed at various temperatures.

At $\Delta T=(180 \div 360)^{\circ} \mathrm{C}$, the growth rate of $W$-parameter decreases. $W$-parameter reached the maximum value in recrystallized samples (Fig. 4).

Figure 5 shows that data points of $S=f(W)$ plot can generate two straight line segments with different inclination to the axes. This enables the determination of two values of $R$-parameter indicating the difference of defect types prevailing as positron traps in SMC nickel at annealing temperatures $\Delta T=(20 \div 180)^{\circ} \mathrm{C}\left(R_{1}\right)$ and $\Delta T=(180 \div 360)^{\circ} \mathrm{C}\left(R_{2}\right)$.

Positrons are trapped at vacancy in metal if average distance between them is smaller than mean free path of thermalized positrons [11]. According to [7], the mean positron diffusion length in nickel at room temperature is $l_{\text {term }}=150 \mathrm{~nm}$. STM results of SMC nickel showed the subgrain growth within the limits of $\Delta l=(35 \div 60) \mathrm{nm}$ at the temperature range $\Delta T=$ $(20 \div 180)^{\circ} \mathrm{C}$ (Fig. 2b). Thus, the main positron traps with the component $\tau_{2}=165 \mathrm{ps}$ of PLS in the temperature range $\Delta T=(20 \div 180)^{\circ} \mathrm{C}$ (Fig. 3$)$ are LAB. This also allows one to explain the growth of $W$-parameter, which testifies chemical environment change at the site 
of positron annihilation (Fig. 5). Apparently, LAB chemical composition alters due to entrainment of impurities from bulk crystallite during subgrain growth.

The component $\tau_{2}=150 \mathrm{ps}$ and $\tau_{3}=(230 \div 290) \mathrm{ps}$ at the $T>180^{\circ} \mathrm{C}$ indicates positrons trap at dislocation and vacancy cluster correspondingly. This allows to conclude that dominant centers of positron trapping in SMC nickel at $T \geq 240{ }^{\circ} \mathrm{C}$ are dislocation left in nickel after in situ recrystallization.

\section{Conclusions}

1. Several processes have been revealed using STM in SMC nickel produced by ECAP during heating within the temperature range $\Delta T=(20 \div 180){ }^{\circ} \mathrm{C}$ : (a) lower non-equiaxiality of grains, (b) grain refinement, (c) subgrain growth. Annealing above $180^{\circ} \mathrm{C}$ leads to grain growth, probably, due to in situ recrystallization.

2. The dominant centers of positron trapping at $\Delta T=$ $(20 \div 180)^{\circ} \mathrm{C}$ are LAB enriched by impurities. Subgrain growth due to LAB displacement is accompanied by alteration of their chemical composition owing to entrainment of impurities from bulk crystallites, which causes the change of the $W$ and $R$ parameters.

3. The dominant positron trapping centers at temperatures of $\Delta T=(180 \div 360)^{\circ} \mathrm{C}$ are dislocations left in nickel after recrystallization in situ.

4. Vacancy clusters show high stability in SMC nickel structure. With increasing temperature within the range of $\Delta T=(20 \div 300)^{\circ} \mathrm{C}$, the size of clusters varies from 4 to 9 vacancies.

\section{References}

[1] R.Z. Valiev, I.V. Alexandrov, Nanostructured Materials Produced by Severe Plastic Deformation, Logos, Moscow 2000.

[2] T.G. Langdon, Acta Mater. 61, 7035 (2013).

[3] P.V. Kuznetsov, I.V. Petrakova, T.V. Rakhmatulina, A.A. Baturin, A.V. Korznikov, Industr. Lab. 78, 26 (2012).

[4] Z.Q. Yang, Mater. Lett. 60, 3846 (2006).

[5] R.S. Laptev, Y.S. Bordulev, V.N. Kudiyarov, A.M. Lider, G.V. Garanin, Adv. Mater. Res. 880, 134 (2014)

[6] S.V. Divinski, G. Reglitz, M. Wegner, M. Peterlechner, G. Wilde, J. Appl. Phys. 115, 113503 (2014).

[7] J. Cizek, I. Prochazka, M. Cieslar, I. Stulikova, F. Chmelik, R. Islamgaliev, Phys. Status Solidi A 191, 391 (2002).

[8] T.E.M. Staab, R. Krause-Rehberg, B. Kieback, J. Mater. Sci. 34, 3833 (1999).

[9] G. Dlubekt, O. Brummert, N. Meyendorfi, P. Hautojarvi, A. Vehanent, J. Yli-Kauppila, J. Phys. F Met. Phys. 9, 1961 (1979).

[10] M.J. Puska, R.M. Nieminen, J. Phys. F Met. Phys. 13, 333 (1983).

[11] R. Krause-Rehberg, H.S. Leipner, Positron Annihilation in Semiconductors: Defect Studies, Springer, Berlin 1999.

[12] L. Liszkay, C. Corbel, L. Baroux, P. Hautojarvi, M. Bayhan, A.W. Brinkman, S. Tatarenko, Appl. Phys. Lett. 64, 1380 (1994). 